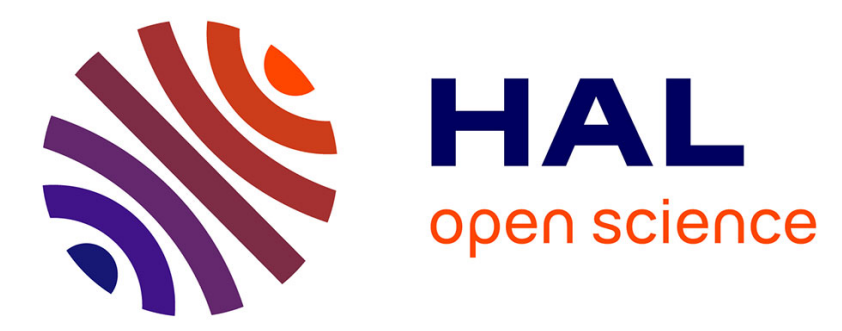

\title{
Processus catalytiques dans un réacteur à plasma hors d'équilibre I. Fixation de l'azote dans le système $\mathrm{N} 2-\mathrm{CH} 4$
}

D. Rapakoulias, J. Amouroux

\section{- To cite this version:}

D. Rapakoulias, J. Amouroux. Processus catalytiques dans un réacteur à plasma hors d'équilibre I. Fixation de l'azote dans le système N2-CH4. Revue de Physique Appliquée, 1980, 15 (7), pp.1251-1259. 10.1051/rphysap:019800015070125100 . jpa-00244847

\section{HAL Id: jpa-00244847 https://hal.science/jpa-00244847}

Submitted on 1 Jan 1980

HAL is a multi-disciplinary open access archive for the deposit and dissemination of scientific research documents, whether they are published or not. The documents may come from teaching and research institutions in France or abroad, or from public or private research centers.
L'archive ouverte pluridisciplinaire HAL, est destinée au dépôt et à la diffusion de documents scientifiques de niveau recherche, publiés ou non, émanant des établissements d'enseignement et de recherche français ou étrangers, des laboratoires publics ou privés. 


\title{
Processus catalytiques dans un réacteur à plasma hors d'équilibre I. Fixation de l'azote dans le système $\mathrm{N}_{2}-\mathrm{CH}_{4}$
}

\author{
D. Rapakoulias et J. Amouroux \\ Laboratoire de Génie Chimique, E.N.S.C.P., 11, rue Pierre-et-Marie-Curie \\ 75231 Paris Cedex 05, France
}

(Reçu le 23 janvier 1980, révisé le 24 mars 1980, accepté le 1 er avril 1980)

\begin{abstract}
Résumé. - Une augmentation importante du taux de fixation de l'azote est observée en présence d'une grille métallique $(\mathrm{Fe}, \mathrm{Cu}, \mathrm{Ta}, \mathrm{W}, \mathrm{Mo})$ dans le réacteur. L'expérience montre qu'il s'agit d'un processus hétérogène, favorisé par une forte excitation vibrationnelle des réactifs. Dans le mécanisme proposé les métastables moléculaires (essentiellement $\mathrm{A}^{3} \Sigma_{\mathrm{u}}$ ) ou le fondamental de $\mathrm{N}_{2}$, fortement excités vibrationnellement par la décharge, acquièrent par chimisorption sur le métal, le complément d'énergie nécessaire à leur dissociation. L'influence de la nature hors d'équilibre du plasma est aussi mise en évidence.
\end{abstract}

\begin{abstract}
A significant improvement of Nitrogen fixation was observed in presence of a metallic grid ( $\mathrm{Fe}, \mathrm{Cu}$, W, Ta, Mo) in the reactor. Experience showed that it is a heterogeneous process favored by a high vibrational excitation of the reactants. A catalysis mecanism is proposed in which the lowest molecular metastable or the fundamental of $\mathrm{N}_{2}$, vibrationally excited by the discharge, gain by chemisorption on the metal the supplement of energy necessary to their dissociation. So the catalytic process studied allows the utilisation for chemical reactivity of the important vibrational excitation of $\mathrm{N}_{2}$ molecule in the low pressure plasma chemical reactors.
\end{abstract}

Introduction. - L'excitation des réactifs dans un plasma basse pression n'est pas sélective et le rendement énergétique du réacteur est généralement faible.

En général le mauvais rendement énergétique du réacteur est dû à la consommation d'une grande partie de l'énergie induite par des états faiblement excités qui ne participent pas à la réactivité chimique.

Il paraît donc nécessaire de rechercher des catalyseurs permettant d'améliorer le rendement et la sélectivité du réacteur.

Jusqu'à aujourd'hui peu de travaux ont été consacrés à l'étude de la catalyse en phase plasma. Dans le domaine de la catalyse homogène, Kaufman et Kelso [1] ont étudié en 1960 la dissociation de $\mathrm{O}_{2}$, en présence de $\mathrm{N}_{2}, \mathrm{NO}, \mathrm{Ar}, \mathrm{He}, \mathrm{CO}_{2}$. Seuls les deux premiers gaz ont permis une augmentation du rendement. En 1964, Young et coll. [2, 3] étudient l'effet de $\mathrm{O}_{2}$ et $\mathrm{SF}_{6}$ sur la dissociation de $\mathrm{N}_{2}$ dans une décharge de micro-ondes, tandis que Safrany et coll. [4] étudient l'effet de HCN. Des effets de catalyse sont détectés. Enfin, Winkler et coll. [5] réalisent en 1971 l'étude de l'effet catalytique d'un grand nombre de gaz sur la dissociation de $\mathrm{N}_{2}$ dans une décharge de micro-ondes, et proposent un mécanisme de catalyse complexe, basé sur le ralentissement des électrons libres et l'absorption par le catalyseur des électrons lents.
En catalyse hétérogène, une première expérience est réalisée en 1974 par Brooks et Seaport [6] dans une décharge micro-ondes de $\mathrm{NH}_{3}$ et $\mathrm{C}_{6} \mathrm{H}_{6}$. Le catalyseur (toile de $\mathrm{Ni}$ ) provoque la diminution de la production de $\mathrm{C}_{2} \mathrm{H}_{2}$ au profit de l'aniline. La complexité du système réactionnel ne permet pas aux auteurs d'établir un mécanisme de catalyse. L'année suivante, P. Mœubus [7, 8] étudie l'effet catalytique du fer sur le craquage du propane dans un plasma d'argon, à pression atmosphérique. L'effet observé (augmentation de la conversion en $\mathrm{C}_{2} \mathrm{H}_{2}$ ) est expliqué soit par un mécanisme hétérogène (absorption sur le fer condensé) soit par un mécanisme homogène (photosensibilisation) qui stabilise le produit recherché $\mathrm{C}_{2} \mathrm{H}_{2}$ ou ses précurseurs $\left(\mathrm{C}_{2} \mathrm{H}\right)$.

Un travail plus suivi dans ce domaine est réalisé en Union Soviétique par l'équipe de E. N. Eremin [9-11] qui, depuis 1944, étudie dans une décharge directe/basse pression l'influence de plusieurs métaux, tels que $\mathrm{Fe}, \mathrm{Ag}, \mathrm{Cu}, \mathrm{Pt}, \mathrm{Pd}$, Ni sur le taux de décomposition de $\mathrm{NH}_{3}$ et sur la formation d'oxydes d'azote à partir de leurs éléments. D'autres solides $(\mathrm{NaOH}$, $\mathrm{KOH}, \mathrm{CuO}$ ) ont aussi été employés comme catalyseurs. L'augmentation du rendement chimique constatée atteint jusqu'à 20 fois le rendement sans catalyseur. Le mécanisme proposé est basé sur des processus physicochimiques à la surface de la cathode 
et ne peut donc pas être appliqué au cas de la décharge H.F.

Le petit nombre et la disparité des systèmes étudiés n'a pas permis jusqu'ici d'aboutir à une connaissance systématique de la catalyse en phase plasma. Dans notre travail nous allons donc rechercher tout d'abord à établir l'existence de phénomènes catalytiques de la fixation de $\mathrm{N}_{2}$, dus à la présence de métaux dans notre réacteur. Ensuite nous proposons un mécanisme de la catalyse à partir de l'analyse spectroscopique du plasma et de l'étude de sa réactivité chimique en l'absence de catalyseur, ce qui nous permet d'étudier le rôle de l'excitation vibrationnelle qui est, rappelonsle, la principale forme d'excitation dans les plasmas à pression réduite.

1. Dispositif expérimental et mode opératoire. Le dispositif expérimental a été décrit dans un article antérieur [12] : un plasma d'azote est créé par une décharge H.F. (40 $\mathrm{MHz}$ ) à pression réduite (5 à 40 mbar). Ce plasma rencontre au niveau de la paroi de trempe un flux de $\mathrm{CH}_{4}$ froid à contre-courant (Fig. 1). La zone de mélange des deux gaz est ainsi à la fois la zone réactionnelle et la zone de trempe. Le catalyseur est introduit dans cette zone (Fig. 2). Les gaz sortant du réacteur sont analysés par chromatographie en phase gazeuse [13].

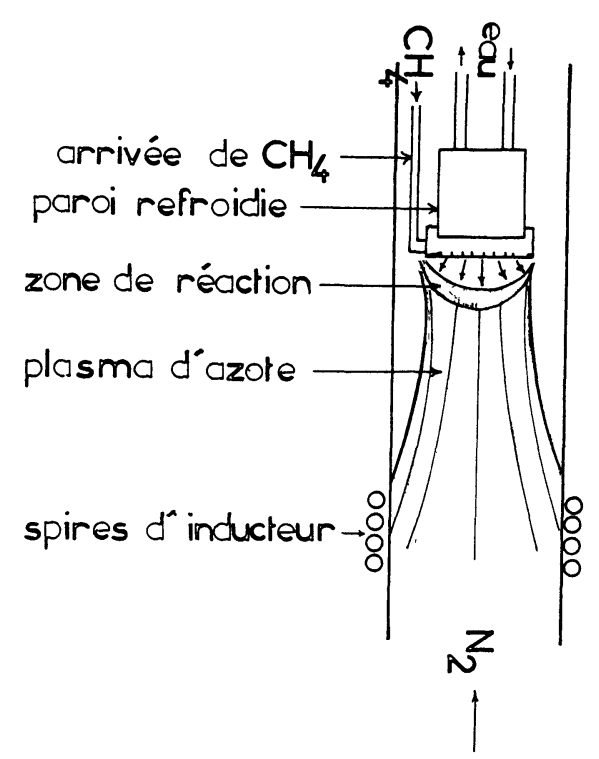

Fig. 1. - Schéma du réacteur sans catalyseur.

[Reactor without catalyst.]

Le catalyseur est constitué d'un assemblage de fils métalliques formant une grille et soutenus par un support en acier inoxydable fixé sur la paroi. Il peut être placé en différentes positions à l'intérieur du

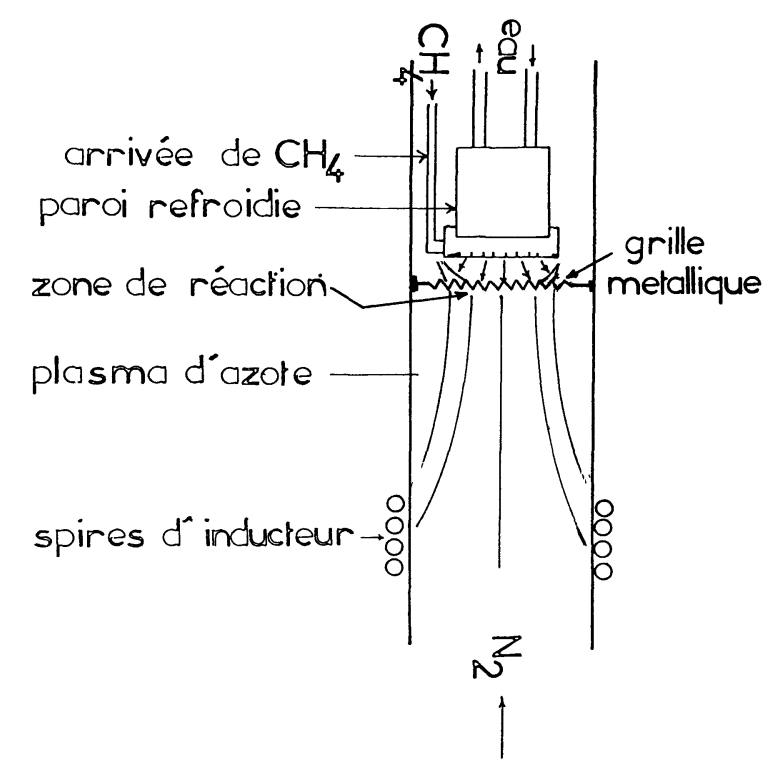

Fig. 2. - Schéma du réacteur avec la grille de catalyseur.

[Reactor with metal grid as catalyst.]

réacteur par modification de la position du support.

La présence de cette grille modifie légèrement la forme du plasma : la zone réactionnelle change de forme (Fig. 2) et une trempe partielle de la torche est observée. Une légère évaporation du métal est parfois observée.

Les métaux testés comme catalyseurs sont les $\mathrm{Fe}$, $\mathrm{Cu}, \mathrm{Mo}, \mathrm{Ta}, \mathrm{W}$. La plupart des essais ont été réalisés avec le Mo, qui semble être efficace. Les métaux à bas point de fusion $(\mathrm{Fe}, \mathrm{Cu})$ fondent parfois au cours de l'expérience.

Pour détecter les phénomènes catalytiques, chaque expérience est effectuée deux fois : une fois sans catalyseur et l'autre fois en sa présence, et nous comparons ensuite les taux de fixation de l'azote et la sélectivité vis-à-vis du HCN. Le taux de fixation est défini en $\%$ de l'azote initial sous forme de HCN. La sélectivité du réacteur $S$ est définie par la relation

$$
S=\frac{\% \text { du carbone transformé en } \mathrm{HCN}}{\% \text { du carbone transformé en } \mathrm{C}_{2} \mathrm{H}_{2}}
$$

2. Rappel des conclusions de l'étude du réacteur sans catalyse. - Dans une étude précédente [14] nous avons analysé le système réactionnel sans catalyse. Parmi les conclusions de cette étude, il nous sera utile d'en rappeler les suivants :

a) La molécule d'azote, excitée dans la décharge produit trois catégories d'états excités : les états très excités dissociatifs $\left(\mathrm{N}_{2}^{* *}\right)$, les états modérément excités $\left(\mathbf{N}_{2}^{*}\right)$ et les états peu excités $\left(\mathbf{N}_{2}^{\prime}\right)$ ne participant à aucune réaction. 


$$
\mathrm{N}_{2}+\mathrm{e}_{\mathrm{N}_{2}^{\prime}+\mathrm{N}_{2}^{*}+\mathrm{CH}_{4} \stackrel{k_{2}}{\longrightarrow} \mathrm{C}_{2} \mathrm{H}_{2}}^{\longrightarrow} \longrightarrow
$$

b) L'état dissociatif désigné par $\mathrm{N}_{2}^{* *}$ est formé essentiellement par les états électroniques $\mathrm{C}^{3} \Pi_{\mathrm{u}}$ et et $\mathrm{B}^{3} \Pi_{\mathrm{g}}$ fortement excités vibrationnellement (prédissociation). La participation des atomes d'azote et de l'ion $\mathrm{N}_{2}^{+}$à la fixation est négligeable.

c) L'état $\mathrm{N}_{2}^{*}$ responsable du transfert d'excitation aboutissant à la formation de $\mathrm{C}_{2} \mathrm{H}_{2}$ est l'état métastable $\mathrm{A}^{3} \Sigma_{\mathrm{u}}^{+}$.

d) La population des états excités décroît dans l'ordre $\mathrm{N}_{2}^{\prime}>\mathrm{N}_{2}^{*}>\mathrm{N}_{2}^{* *}$.

e) La constante de vitesse de la fixation $\left(k_{1}\right)$ est très supérieure à la constante du transfert d'énergie $\left(k_{2}\right)$ (au moins deux ordres de grandeur).

3. Résultats. - 3.1 MiSE EN ÉVIDENCE DU PHÉNOMÈNE CATALYTIQUE DANS UN RÉACTEUR A CONTRECOURANT. - Nous avons d'abord cherché à établir l'existence d'un processus catalytique par la comparaison des produits de la réaction avec et sans grille métallique.

En faisant varier l'énergie induite dans le plasma les résultats montrent (Figs. 3, 4, 5) une augmentation du taux de HCN en présence du métal, tandis que le

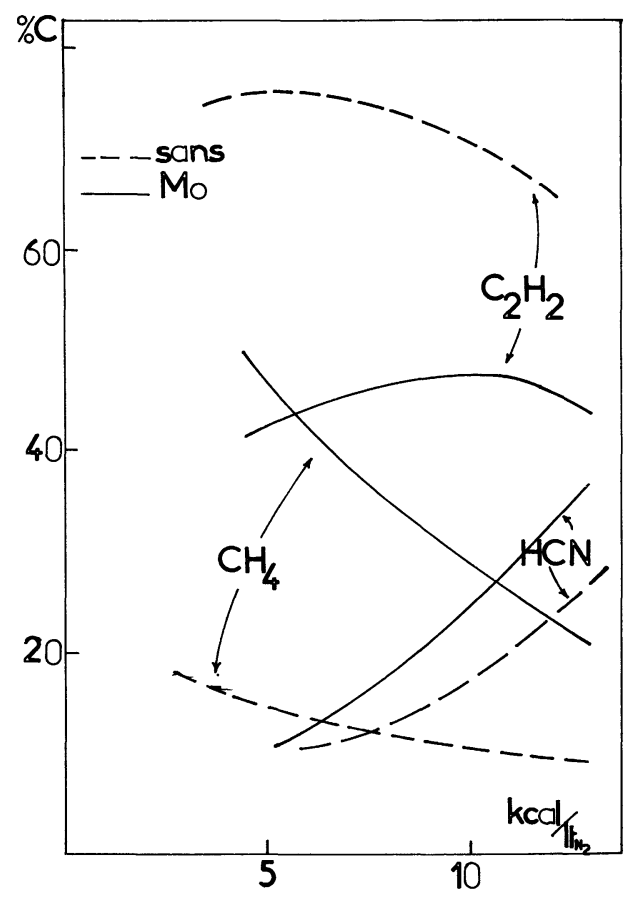

Fig. 3. - Comparaison des produits de la réaction avec et sans catalyseur.

[Comparison of reactor's products in presence and in absence of catalyst.] (fixation, $E \geqslant 9,7 \mathrm{eV}$ )

(transfert d'excitation 9,7 $\geqslant E \geqslant 4,6 \mathrm{eV}$ )

pas de réaction $(E \leqslant 4,6 \mathrm{eV})$.

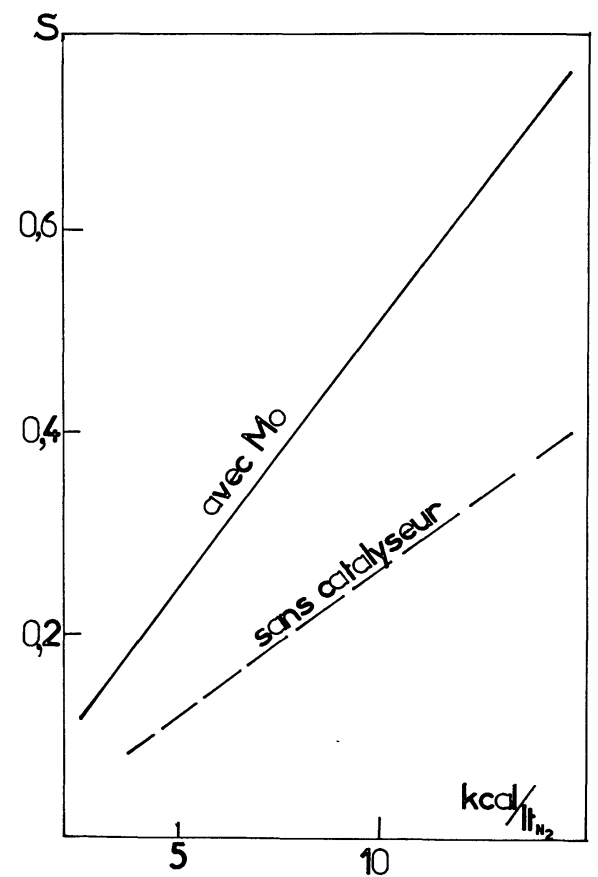

Fig. 4. - Sélectivité du réacteur vis-à-vis du $\mathrm{HCN}$ avec et sans catalyseur.

[Reactors selectivity for $\mathrm{HCN}$ with and without catalyst.]

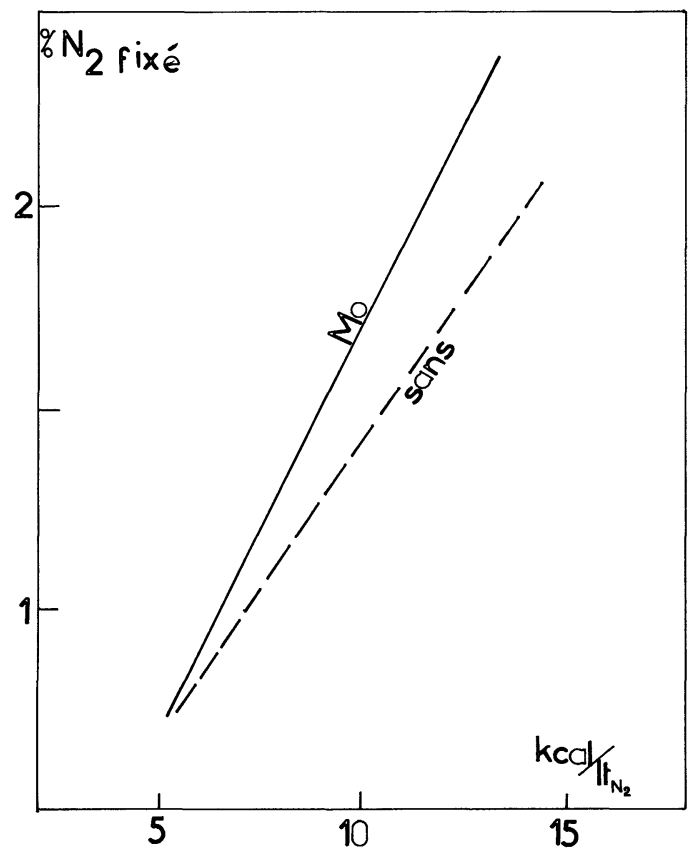

Fig. 5. - Taux de fixation de l'azote en présence et en absence de catalyseur.

[Rate of nitrogen fixation in presence and in absence of catalyst.] 
taux de $\mathrm{C}_{2} \mathrm{H}_{2}$ décroît. Le $\mathrm{CH}_{4}$ non réagi crô̂t aussi en présence de la grille métallique.

Des résultats analogues ont été obtenus en faisant varier le débit de $\mathrm{CH}_{4}$ (Fig. 6) et le débit de $\mathrm{N}_{2}$ (Fig. 7) en présence d'autres métaux $(\mathrm{Fe}, \mathrm{Cu})$.

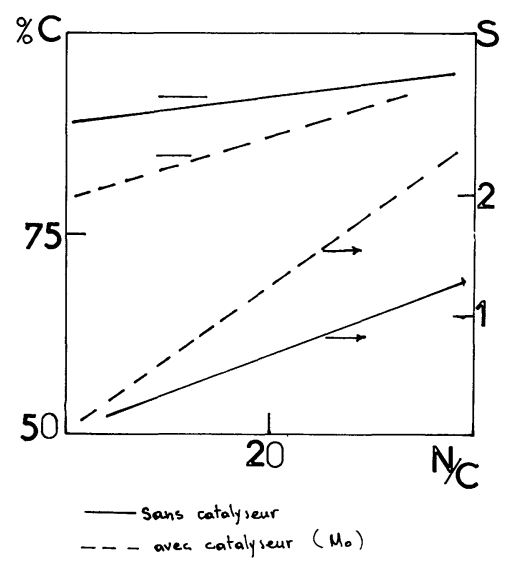

Fig. 6. - Variation du taux de transformation de carbone et de la sélectivité du réacteur en fonction du débit de $\mathrm{CH}_{4}$ (exprimé en rapport $\mathrm{N} / \mathrm{C}$ ).

[Carbon conversion rate and selectivity as a function of $\mathrm{CH}_{4}$ flow rate (expressed as $\mathrm{N} / \mathrm{C}$ ratio).]

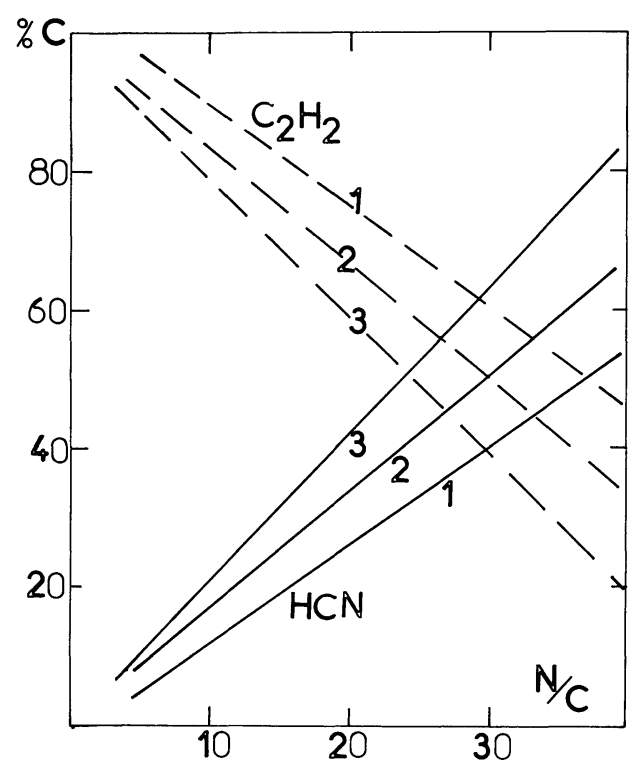

Fig. 7. $-\%$ du carbone transformé, converti en $\mathrm{C}_{2} \mathrm{H}_{2}$ (pointillés) et en $\mathrm{HCN}$ (trait plein), en fonction du rapport $\mathrm{N} / \mathrm{C}$. Puissance induite constante : 2,4 kV-0,83 A. Débit $\mathrm{CH}_{4}$ stable. Débit $\mathrm{N}_{2}$ varié. 1 : sans catalyseur ; 2 : grille du $\mathrm{Cu} ; 3:$ grille de Fe.

$\left[\%\right.$ carbon conversion as a function of $\mathrm{N}_{2}$ flow rate (expressed as $\mathrm{N} / \mathrm{C}$ ratio).]

Nous pouvons ainsi conclure que la présence d'une grille métallique dans le réacteur produit :

a) une diminution du taux de transformation de $\mathrm{CH}_{4}$, par modification du transfert d'énergie entre les deux gaz; b) une diminution de l'efficacité du transfert d'excitation azote méthane (c'est-à-dire de $\mathrm{C}_{2} \mathrm{H}_{2}$ ) ;

c) une augmentation de la fixation de l'azote, associée à une sélectivité du réacteur vis-à-vis du $\mathrm{HCN}$ (Figs. 4, 5).

Ces conclusions établissent sans équivoque la présence d'un phénomène catalytique. En effet, si le rôle de la grille métallique se limitait à une simple modification du contact entre les gaz, le taux de $\mathrm{C}_{2} \mathrm{H}_{2}$ et de $\mathrm{HCN}$ auraient dû baisser simultanément.

\subsection{NATURE DU PHÉNOMÈNE CATAlytiQUe. - Après} avoir détecté le processus catalytique dû à la présence d'un métal dans le réacteur, nous avons cherché à établir si il s'agissait d'un processus homogène en phase gazeuse (gaz-vapeur métallique) ou d'un processus hétérogène (gaz-solide).

Pour cela nous avons conduit deux séries d'expériences : dans la première, nous avons fait varier la position de la grille métallique par rapport à la zone réactionnelle. En effet trois cas sont possibles : la grille se trouve au-dessus, dedans ou en dessous de la zone réactionnelle (Figs. $8 a, b, c$ respectivement). Comme mesure de la position de la grille nous avons utilisé la distance grille-paroi de trempe. Les autres paramètres expérimentaux (pression, énergie induite, débits) sont maintenus constants. Les résultats de l'expérience sont portés sur la figure 9. Quand la grille de catalyseur est au-dessus de la zone réactionnelle $(d<1 \mathrm{~cm})$ sa présence n'a aucun effet. Lorsque elle est dans la zone réactionnelle $(2<d<4 \mathrm{~cm})$ le taux de fixation de $\mathrm{N}_{2}$ augmente par rapport aux expériences sans catalyseur, en passant par un maximum. Enfin, lorsque la grille s'éloigne de la zone réactionnelle $(d>4 \mathrm{~m})$ le taux de fixation chute.

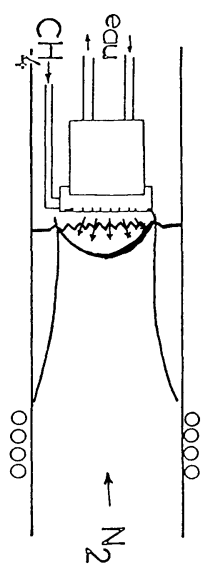

$(\bar{a})$

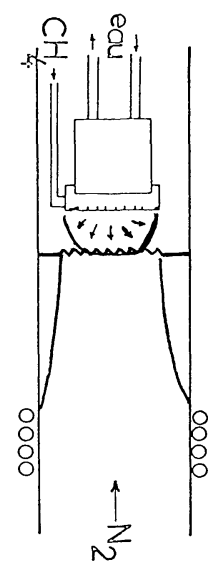

(b)

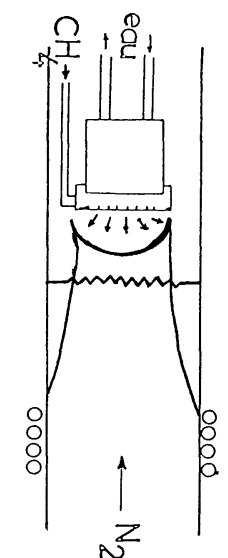

(e)
Fig. 8. - Positions de la grille du catalyseur par rapport à la zone réactionnelle. (a) Grille au-dessus de la zone réactionnelle; $(b)$ grille au niveau de la zone réactionnelle; $(c)$ grille au-dessous de la zone réactionnelle.

[Positions of catalyst grid relative to reaction zone : (a) grid above reaction zone; $(b)$ grid into reaction zone; $(c)$ grid below reaction zone.] 


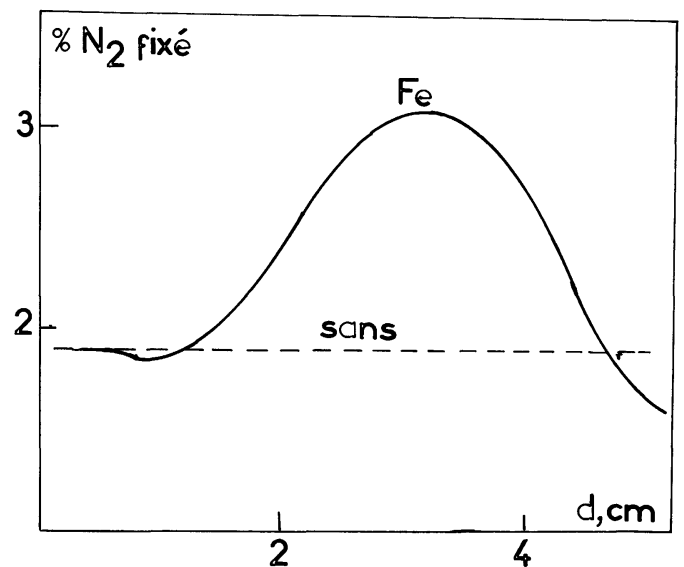

Fig. 9. - Variation du taux de fixation de l'azote avec la position de la grille de catalyseur dans le réacteur. $d=$ distance entre la grille et la paroi de trempe.

[Variation of nitrogen fixation rate in function of grid position in the reaction zone. $d$ : distance between the grid and the quenching wall.]

Cette influence de la position de la grille indique la présence d'un processus de catalyse sur la surface du métal. En effet s'il s'agissait d'un phénomène en phase gazeuse (catalyse par les vapeurs métalliques) l'effet catalytique ne dépendrait pas notablement de la position de la grille par rapport à la zone réactionnelle, car la vaporisation du métal a la même importance quelle que soit la position de la grille. Pour confirmer cette hypothèse nous avons effectué des essais en plongeant un seul fil de Fe (dont la vapo-

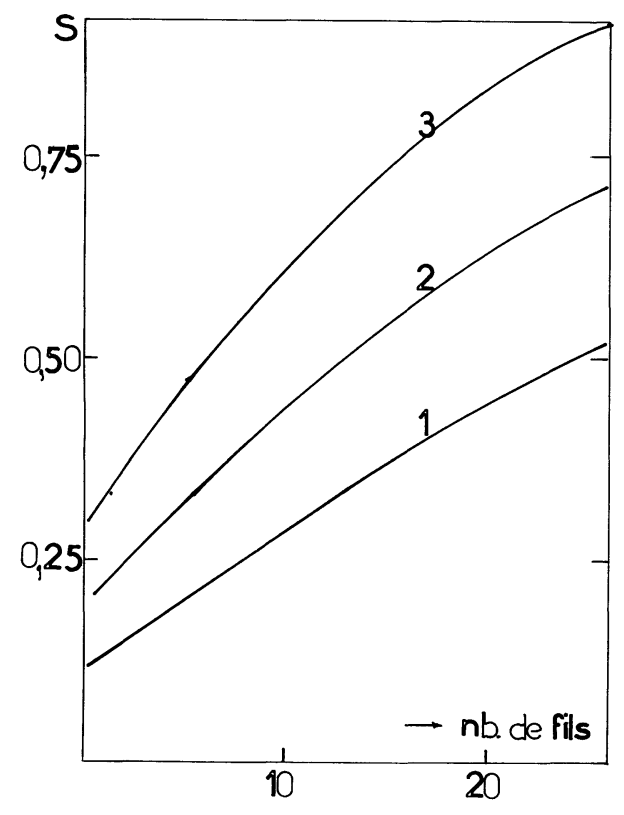

Fig. 10. - Variation de la sélectivité du réacteur en fonction de la surface (nombre de fils) du catalyseur, pour différentes valeurs de l'énergie induite. (Pression, débits constants.)

[Reactor's selectivity as a function of catalyst surface (number of wires in the grid), for three different values of induced energy (pressure and flow rates are constant).] risation est importante) au centre du plasma de $\mathrm{N}_{2}$, sans employer de grille. Aucun effet catalytique ne fut observé dans ce cas.

Dans la deuxième série d'expériences nous avons cherché à démontrer la présence d'un processus catalytique hétérogène en faisant varier le nombre de fils métalliques de la grille, c'est-à-dire la surface du catalyseur. La grille est posée au niveau de la zone réactionnelle. Les autres paramètres expérimentaux sont maintenus constants. Les résultats sont portés sur la figure 10 .

La sélectivité du réacteur (Fig. 10) augmente quand le nombre de fils passe de 0 à 26 . Mais lorsque la grille devient plus dense, la modification du contact gaz-gaz produit progressivement une diminution de la sélectivité.

En conclusion, ces résultats montrent que l'effet catalytique augmente avec la surface du catalyseur, ce qui confirme le caractère hétérogène du phénomène. Toutefois du fait de l'introduction des gaz à contrecourant, lorsque la grille du catalyseur devient trop dense, elle provoque une diminution du contact entre les réactifs. Le résultat global est donc un compromis entre ces deux tendances.

4. Interprétation du mécanisme de catalyse. 4.1 Notion GÉNÉRALE ET VOIE POSSIBLE DE LA CATALYSE EN PHASE PLASMA. - En chimie, la théorie des collisions, appliquée au cas d'une réaction bimoléculaire

$$
\mathrm{A}+\mathrm{BC} \rightarrow \mathrm{AB}+\mathrm{C}
$$

permet d'établir une constante de vitesse $k$ selon l'expression $[15,16]$ :

$$
k=P \pi \sigma_{\mathrm{m}}^{2}\left(\frac{8 R T}{\pi} \times \frac{m_{\mathrm{A}}+m_{\mathrm{BC}}}{m_{\mathrm{A}} m_{\mathrm{BC}}}\right)^{1 / 2} \rho^{-E / R T}
$$

où $P$ est le facteur stérique ou facteur de probabilité, $\sigma_{\mathrm{m}}$ est la section de capture des réactifs,

$E$ est l'énergie d'activation de la réaction,

$m_{\mathrm{A}}, m_{\mathrm{BC}}$ la masse moléculaire des réactifs.

Le rôle d'un catalyseur est d'augmenter la vitesse de la réaction, c'est-à-dire la constante de la vitesse. Ceci peut être obtenu par action sur le facteur de probabilité (augmentation de la durée de vie de l'espèce active) ou sur la section de capture des réactifs (formation d'un complexe) ou par abaissement de l'énergie d'activation (Fig. 11). Mais, en aucun cas la catalyse ne pourra changer la concentration finale des produits, car elle active au même degré la réaction directe et la réaction inverse.

En phase plasma, la réaction chimique suit une trajectoire plus complexe, caractérisée par des étapes hors d'équilibre que nous avons analysées dans l'article précédent [14].

La réaction a lieu à partir des états très excités et ainsi la vitesse de réaction est très grande par rapport 


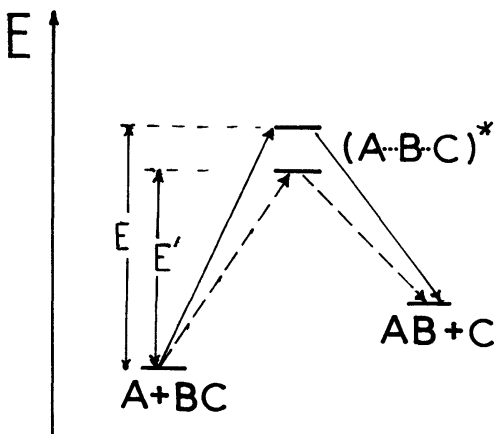

temps

Fig. 11. - Catalyse par abaissement de l'énergie d'activation.

[Catalysis in a conventional chemical reaction.]

au temps de séjour dans le réacteur. Un processus de catalyse dans le sens classique du terme n'a pas de signification pratique.

Nous pouvons par contre espérer augmenter, par la présence d'un tiers corps, le rendement final des produits de la réaction.

En effet, lorsque la réaction précédente

$$
\mathrm{A}+\mathrm{BC} \rightarrow \mathrm{AB}+\mathrm{C}
$$

a lieu dans un réacteur à plasma hors d'équilibre, trois étapes principales peuvent être distinguées (Fig. 12).

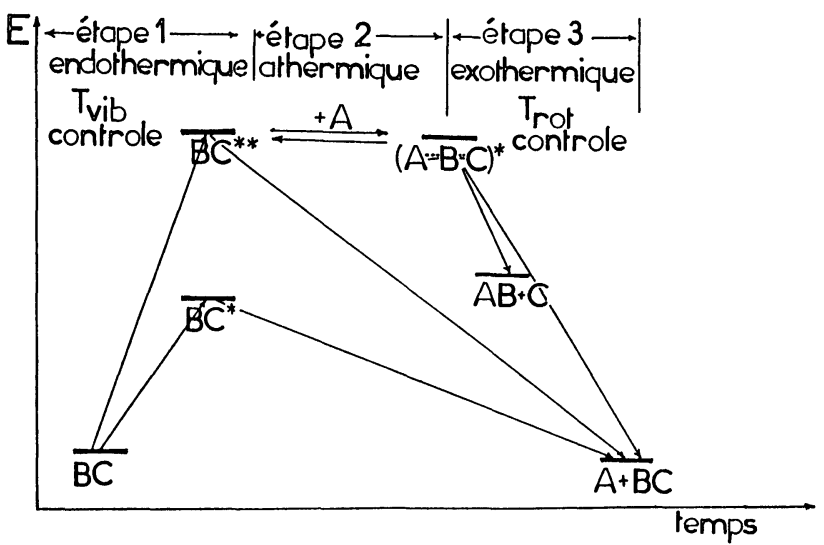

Fig. 12. - Evolution de la réaction en phase plasma $\mathrm{A}+\mathrm{BC}^{* *} \rightarrow$ $\mathrm{AB}+\mathrm{C}$ où seul le réactif $\mathrm{BC}$ est excité dans la décharge.

[Diagram of the plasma-phase reaction $\mathrm{A}+\mathrm{BC}^{* *} \rightarrow \mathrm{AB}+\mathrm{C}$ where only reactant $\mathrm{BC}$ is excited by the discharge.]

Une première étape endothermique d'excitation des réactifs, une seconde étape de recombinaison entre espèces excitées et une troisième étape exothermique de stabilisation des produits excités (trempe) (pour plus de détails sur cette représentation des réactions chimiques en phase plasma voir les références $[13,14])$.
Dans ces conditions le rendement total de la réaction peut être amélioré par quatre voies différentes :

a) Augmentation de la population de l'espèce réactive $\mathrm{BC}^{* *}$ par transfert sélectif au moyen d'un donneur $D^{*}$ (Fig. 13).

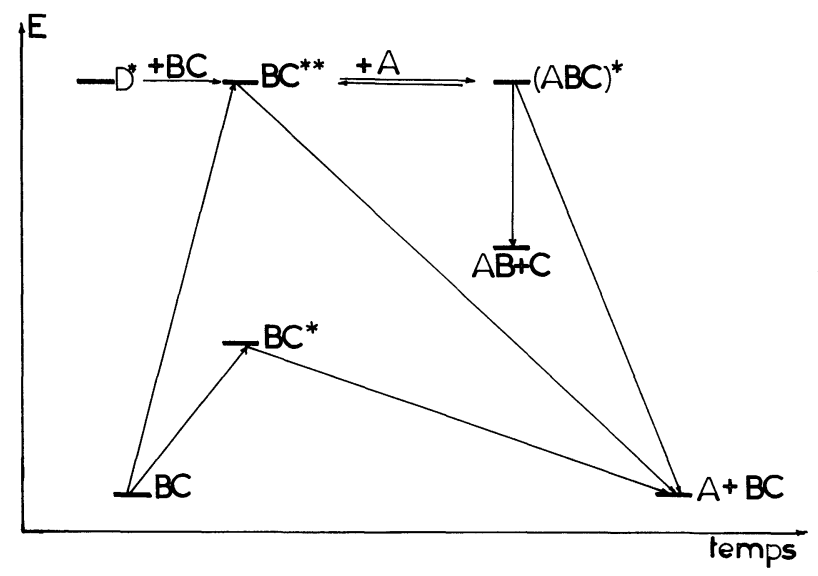

Fig. 13. - Catalyse par transfert sélectif d'énergie vers l'état excité réactif $\mathrm{BC}^{* *}$ au moyen du donneur $D^{*}$.

[Catalysis by selective energy transfert to the reactive excited state $\mathrm{BC}^{* *}$ from a donnor $D^{*}$.]

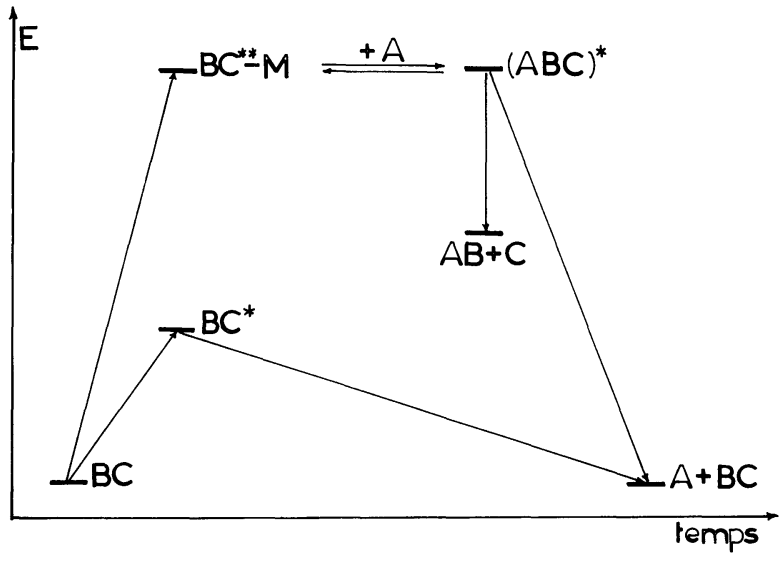

Fig. 14. - Catalyse par stabilisation de l'état excité réactif $\mathrm{BC}^{* *}$. [Catalysis by stabilisation of the reactive excited state $\mathrm{BC}^{* *}$.]

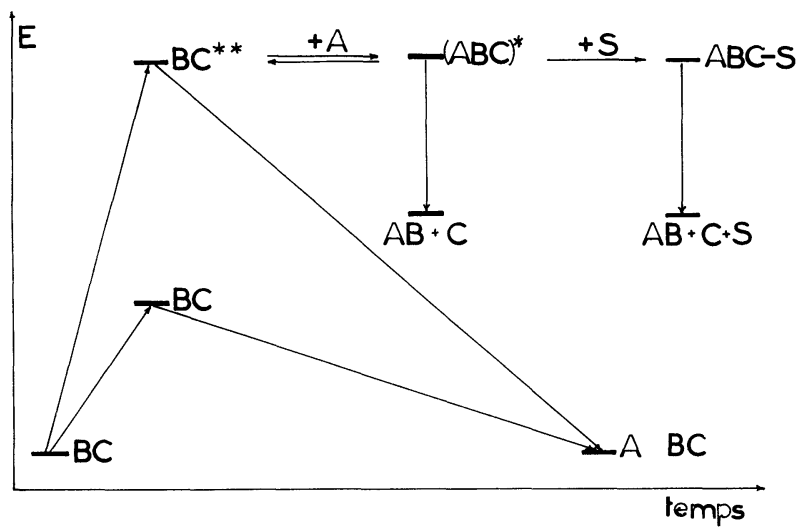

Fig. 15. - Catalyse par stabilisation du produit excité (ABC)* au moyen d'un tiers corps.

[Catalysis by stabilisation of the excited reaction product $(\mathrm{ABC})^{*}$.] 
b) Augmentation de la durée de vie de $\mathrm{BC}^{* *}$ par formation d'un complexe en phase gazeuse ou par absorption sur une surface (Fig. 14).

c) Stabilisation du produit de la réaction (A...B . . C )* par formation de complexes ou désexcitation sur une surface (Fig. 15).

d) Activation de l'état de faible énergie $\mathrm{BC}^{*}$ par chimisorption sur une surface (Fig. 16).

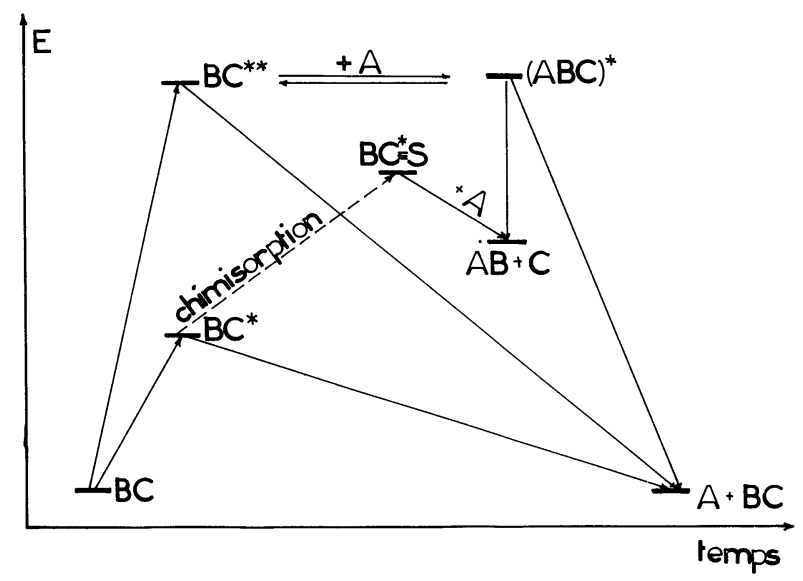

Fig. 16. - Catalyse par chimisorption : l'énergie de l'état $\mathbf{B C}^{*}$ augmente et la réaction avec $\mathrm{A}$ devient possible.

[Catalysis by chemisorption : the energy of the low-lying excited state $\mathrm{BC}^{*}$ is increased and reaction with a becomes possible.]

\subsection{MÉCANISME DE LA CATALYSE DANS NOTRE RÉAC-} TEUR. - 4.2.1 Recherche d'un schéma de catalyse. Cette analyse générale de la catalyse en phase plasma nous aide à élucider le mécanisme de la catalyse dans notre réacteur. En effet, dans nos expériences le cas $(a)$ est exclu, car il n'y a pas de donneur pour un transfert sélectif. La voie $(b)$ semble également inopérante : elle aurait comme conséquence l'augmentation du taux de $\mathrm{HCN}$ sans diminution du taux de $\mathrm{C}_{2} \mathrm{H}_{2}$ (en particulier quand le $\mathrm{CH}_{4}$ est en excès).

Pour examiner la possibilité de catalyse par la voie (c) (désexcitation des produits sur une surface) nous avons établi un diagnostic chimique : nous avons examiné expérimentalement l'influence de la nature du métal sur l'effet catalytique. En effet, la désexcitation d'une molécule sur une surface ne nécessite pas la création de liaisons fortes (chimisorption) entre le métal et la molécule gazeuse, mais seulement un contact physique. Par conséquent, l'effet catalytique ne devrait pas dépendre de la nature du métal [17].

Or, les résultats de l'expérience indiquent une influence marquée de la nature du métal (Fig. 17). Le classement est le suivant (en ordre décroissant).

$$
\mathrm{Mo}>\mathrm{W}>\mathrm{Ta}>\mathrm{Fe}>\mathrm{Cu}\left({ }^{1}\right) \text {. }
$$

Il ne reste donc comme voie possible pour la catalyse que l'activation de l'azote par un processus

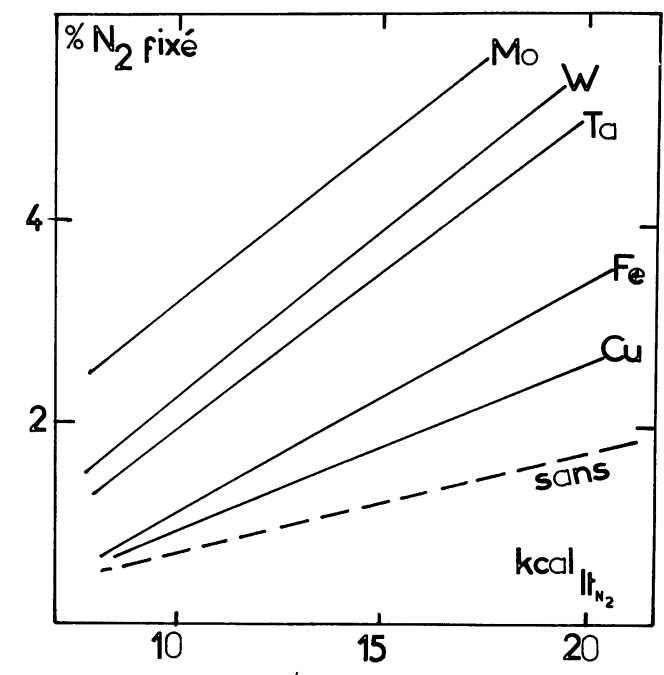

Fig. 17. - Influence de la nature du métal sur l'effet catalytique.

[Influence of metal's nature on the catalytic effect.]

de chimisorption à partir des états excités de faible énergie (voie $(d))$.

Appliqué à notre système réactionnel, ce mécanisme donnerait pour la fixation de $\mathrm{N}_{2}$ le schéma suivant (Fig. 18) :

$$
\begin{array}{r}
\mathrm{N}_{2}^{* *}+\mathrm{CH}_{4} \rightarrow \mathrm{HCN}+\mathrm{H}_{2} \quad \text { (sans catalyse) } \\
\mathrm{N}_{2}^{*} \underset{\text { sur le métal }}{\stackrel{\text { chimisorption }}{\longrightarrow}} \mathrm{N}_{2 \mathrm{ads}}^{* *} \stackrel{+\mathrm{CH}_{4}}{\longrightarrow} \mathrm{HCN}+\mathrm{H}_{2}
\end{array}
$$

(catalyse hétérogène) .

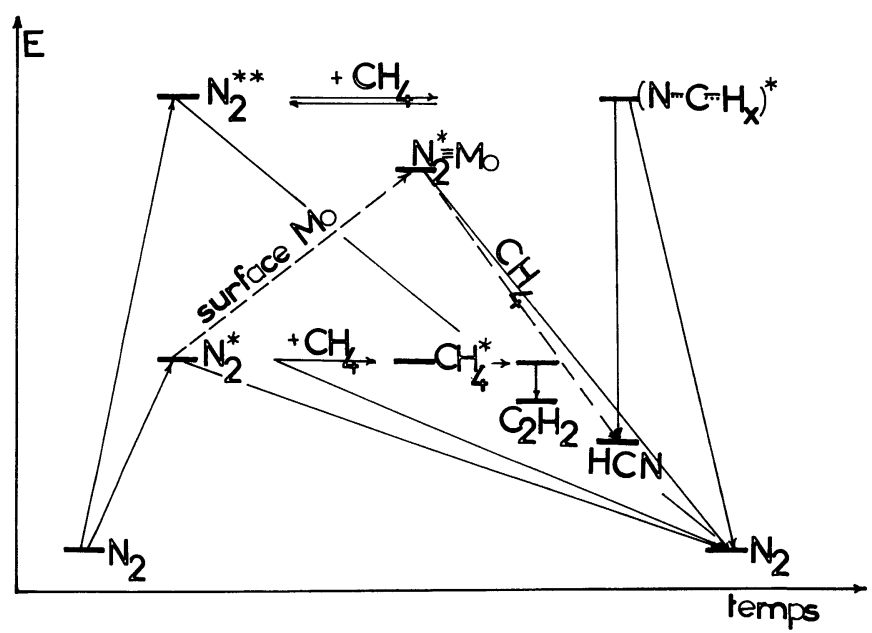

Fig. 18. - Mécanisme de catalyse dans la réaction azote-méthane. [Catalytic mechanism of the nitrogen-methane reaction.]

( $\left.{ }^{1}\right)$ Il est probable, comme l'a montré l'analyse ESCA, que le Mo et le Ta soient oxydés superficiellement. L'analyse par rayons $X$ n'a pas détecté la présence de nitrures sur le catalyseur (après utilisation). 
4.2.2 Confirmation expérimentale du schéma de catalyse proposé. - Ce mécanisme de catalyse est confirmé par les résultats expérimentaux. En effet, il implique une baisse du taux d'acétylène par rapport aux résultats sans catalyse, par suite des réactions simultanées de l'azote faiblement excité avec le méthane soit pour donner $\mathrm{HCN}$, soit $\mathrm{C}_{2} \mathrm{H}_{2}$ :

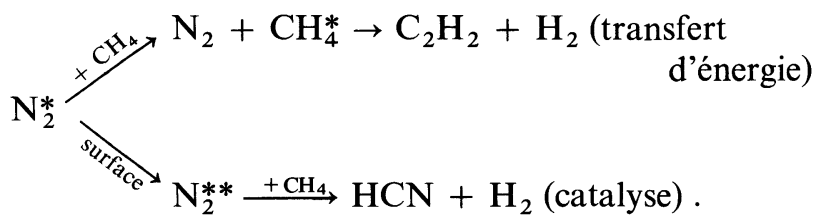

Cette baisse des taux de $\mathrm{C}_{2} \mathrm{H}_{2}$ a été confirmée expérimentalement (Figs. 3 et 7).

4.2.3 Nature de l'état excité responsable de la catalyse. - En ce qui concerne la nature exacte de l'état excité $\mathrm{N}_{2}^{*}$ responsable de la catalyse, les conclusions de l'analyse du réacteur sans catalyse [14] nous fournissent les renseignements nécessaires : la fixation de l'azote se fait à partir de l'état $\mathrm{N}_{2}\left(\mathrm{C}^{3} \Pi_{\mathrm{u}}\right)$, tandis que le transfert d'énergie a lieu à partir du niveau $\mathrm{N}_{2}\left(\mathrm{~A}^{3} \Sigma_{\mathrm{u}}^{+}\right)$fortement excité vibrationnellement.

Ainsi, selon le schéma proposé, la catalyse hétérogène de la fixation de l'azote se fait par chimisorption sur le métal d'une molécule $\mathrm{A}^{3} \Sigma_{\mathrm{u}}^{+}$, excitée à un niveau vibrationnel élevé (Fig. 19b).

L'énergie dégagée lors de la chimisorption rompt la liaison $\mathrm{N}-\mathrm{N}$, déjà fortement affaiblie par l'excitation de la molécule $\mathrm{N}_{2}$ dans la décharge. Les atomes d'azote libérés (Fig. 19c) sont disponibles pour réagir avec le $\mathrm{CH}_{4}$ ou ses fragments et former une molécule instable $\left(\mathrm{N} \ldots \mathrm{C} \ldots \mathrm{H}_{\mathrm{x}}\right)^{*}$. Par désorption de la surface, une molécule HCN stabilisée est formée : en effet, l'énergie nécessaire pour rompre la liaison azote-métal est donnée par la molécule excitée $\left(\mathrm{N} \ldots \mathrm{C} \ldots \mathrm{H}_{\mathrm{x}}\right)^{*}$, qui ainsi se stabilise (Fig. 19d).

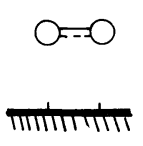

व

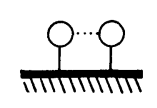

$b$

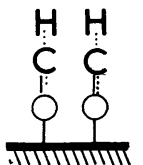

c

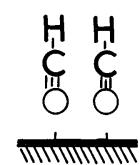

d
Fig. 19. - Catalyse hétérogène de la fixation de l'azote : a) Une molécule excitée (mais pas suffisamment pour se dissocier) s'approche de la surface. $b$ ) Chimisorption de cette molécule sur la surface. L'énergie dégagée rompt la liaison $\mathbf{N}-\mathbf{N}$. c) Sur les. atomes $\mathbf{N}$ adsorbés, les espèces $\mathrm{CH}_{\mathbf{X}}(1<x \leqslant 4)$ viennent réagir et créer ainsi une molécule instable $\left(\mathrm{N} \ldots \mathrm{C} \rightleftharpoons \mathrm{H}_{\mathrm{X}}\right)$. d) Désorption de la molécule $\mathrm{HCN}$ stable : la rupture de la liaison azote-métal absorbe l'énergie contenue dans l'espèce $\left(\mathrm{N} \ldots \mathrm{C} \ldots \mathrm{H}_{\mathrm{X}}\right)$ qui se stabilise sous forme de $\mathrm{HCN}$.

[Heterogeneous catalysis of nitrogen fixation : $a$ ) A weekly excited (non-dissociative) nitrogen molecule approaches the surface. b) Chemisorption of this molecule on the surface. The energy of chemisorption breaks-up the $\mathrm{N}-\mathrm{N}$ bond. $c$ ) Reaction of adsorbed $\mathrm{N}$ atoms with $\mathrm{CH}_{\mathrm{X}}$ molecules $(1<x<4)$. An instable (excited) molecule is formed. $d$ ) The break-up of the metal- $\mathrm{N}$ bond consume the excess energy of this instable molecule and a stable $\mathrm{HCN}$ molecule is formed.]
5. Rôle de la température de vibration dans la catalyse. - Pour conclure ce travail nous avons cherché à établir la relation entre la nature hors d'équilibre du plasma et l'effet catalytique.

L'étude du système sans catalyse a démontré que le taux de fixation de l'azote augmente lorsque la température de vibration augmente (Fig. 20). Cela signifie que l'étape-clé du processus de formation de $\mathrm{HCN}$ est la stabilisation (exothermique) de la molécule intermédiaire excitée (H...C...N)* (Fig. 21).

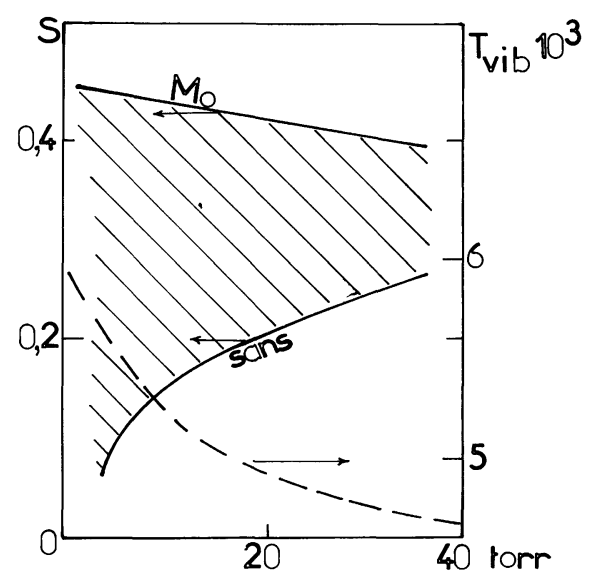

Fig. 20. - Comparaison de la variation de l'étendue de la catalyse (partie hachurée) et de la température de vibration.

[Comparison of the simultaneous variation of catalytic effect (hached surface) and the vibrational temperature.]

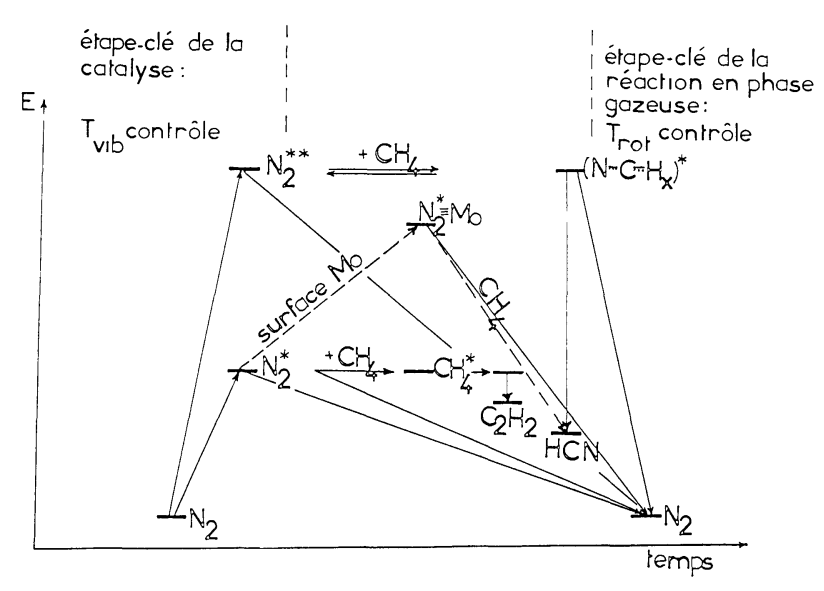

Fig. 21. - Etapes-clés de la fixation de l'azote à partir de l'azote dissociatif (phase gazeuse) et par voie catalytique (réaction de surface).

[Rate-determining steps for nitrogen fixation from dissociative molecules (gaz-phase) and by catalytic way (surface reaction).]

En présence du catalyseur cette relation est inversée : en effet, la mesure simultanée du taux de fixation et de la température de vibration montre que le taux de fixation baisse lorsque la température de vibration baisse (Fig. 20). L'efficacité de la catalyse (partie hachurée de la figure 20) diminue, elle aussi. 
L'étape déterminante du processus catalytique est l'étape endothermique de chimisorption dissociative de la molécule $\mathrm{N}_{2}\left(\mathrm{~A}^{3} \Sigma_{\mathrm{u}}^{+}\right)$sur le métal (Figs. 19b, 21). L'efficacité de cette étape sera d'autant plus grande que l'énergie de vibration est élevée, c'est-à-dire la liaison $\mathrm{N}-\mathrm{N}$ est affaiblie. Par contre, l'étape exothermique de stabilisation de l'intermédiaire $(\mathrm{H} \ldots \mathrm{C} \ldots \mathrm{N})^{*}$ n'est pas déterminante dans le processus catalytique, car l'évacuation de l'excès d'énergie de cet intermédiaire est assurée par la rupture de la liaison carbone-métal lors de la désorption (Fig. 19d).

6. Conclusion. - Au cours de ce travail nous avons tout d'abord mis en évidence expérimentalement l'augmentation du taux de fixation de l'azote et de la sélectivité du réacteur provoquée par la présence d'une grille métallique dans le plasma.

Par l'expérience nous avons ensuite démontré qu'il s'agit d'un phénomène hétérogène gaz-surface du solide, les vapeurs métalliques ne jouant aucun rôle.

La catalyse en phase plasma peut ainsi être définie comme l'augmentation du rendement chimique et énergétique du réacteur provoquée par l'adjonction d'un tiers corps. Elle peut procéder par transfert sélectif vers l'état excité réactif recherché, par augmentation de la durée de vie de cet état, par désexcitation accélérée des produits de la réaction ou par activation supplémentaire des états excités de faible énergie.

Dans notre réacteur la catalyse de la fixation de l'azote se fait par ce dernier mécanisme : le métastable $\mathrm{N}_{2}\left(\mathrm{~A}^{3} \Sigma_{\mathrm{u}}^{+}\right)$ou même le fondamental, déjà fortement excité vibrationnellement par la décharge, acquiert par chimisorption sur la surface du métal le complément d'énergie qui permet la dissociation de la molécule.

Ainsi, ce processus de catalyse permet d'exploiter les états excités de faible énergie (notamment $\mathrm{A}^{3} \Sigma_{\mathrm{u}}^{+}$) de $\mathrm{N}_{2}$ dans le plasma qui, sans catalyse, ne participent pas à la réactivité chimique et constitue la cause principale du faible rendement de la fixation de l'azote dans les plasmas froids.

\section{Bibliographie}

[1] Kaufman, F., Kelso, J. R., J. Chem. Phys. 32 (1960) 301.

[2] Young, R. A., J. Chem. Phys. 60 (1974) 5050.

[3] Young, R. A., Sharpless, R. L., Stringham, R., J. Chem. Phys. 40 (1964) 117.

[4] Safrany, Harteck, Reeves, J. Chem. Phys. 41 (1964) 1161.

[5] Baker, R. R., JaKob, A., Winkler, C. A., Can. J. Chem. 49 (1971) 1671.

[6] Brooks, B. W., Seaport, R. M., J. App. Chem. Biotechnol. 24 (1974) 621

[7] Mceubus, P., J. Electrochem. Soc. 122 (1975) 298.

[8] Mceubus, P., Can. J. Chem. Eng. 53 (1975) 653.

[9] Eremin, E. N., Syaduk, V. L., Russ. J. Phys. Chem. 49 (1975) 587.
[10] Eremin, E. N., Russ. J. Phys. Chem. 48 (1974) 1229.

[11] Eremin, E. N., Borisova, A. N., Russ. J. Phys. Chem. 36 (1962) 1266.

[12] Amouroux, J., Cavvadias, S., Rapakoulias, D., Revue Phys. Appl. 14 (1979) 969.

[13] Rapakoulias, D., Thèse, Université P.-et-M.-Curie, 1979.

[14] Rapakoulias, D., Amouroux, J., Revue Phys. Appl. 14 (1979) 961.

[15] SchaAl, R., La cinétique chimique homogène (P.U.F.) 1971

[16] Ravaille, M., Didier, R., Chimie Générale, Chap. C-5, 1970.

[17] Slater, J. C., Johnson, K. H., Physics Today 27 (1974) 34. 